2014

\title{
Spectroscopy OfThe Inner Companion Of The Pulsar Psr J0337+1715
}

David L. Kaplan

Marten H. van Kerkwijk

Detlev Koester

Ingrid H. Stairs

Scott M. Ransom

See next page for additional authors

Follow this and additional works at: https://researchrepository.wvu.edu/faculty_publications

\section{Digital Commons Citation}

Kaplan, David L.; van Kerkwijk, Marten H.; Koester, Detlev; Stairs, Ingrid H.; Ransom, Scott M.; Archibald, Anne M.; Hessels, Jason W. T.; and Boyles, Jason, "Spectroscopy Of The Inner Companion Of The Pulsar Psr J0337+1715" (2014). Faculty Scholarship. 682. https://researchrepository.wvu.edu/faculty_publications/682 
Authors

David L. Kaplan, Marten H. van Kerkwijk, Detlev Koester, Ingrid H. Stairs, Scott M. Ransom, Anne M.

Archibald, Jason W. T. Hessels, and Jason Boyles 


\title{
SPECTROSCOPY OF THE INNER COMPANION OF THE PULSAR PSR J0337+1715*
}

\author{
David L. Kaplan ${ }^{1}$, Marten H. van Kerkwijk ${ }^{2}$, Detlev Koester ${ }^{3}$, Ingrid H. Stairs ${ }^{4}$, Scott M. Ransom ${ }^{5}$, \\ ANNE M. ARChiBALD ${ }^{6,7}$, Jason W. T. Hessels ${ }^{6,8}$, AND JASON BOYLES $^{9,10}$ \\ ${ }^{1}$ Department of Physics, University of Wisconsin-Milwaukee, 1900 E. Kenwood Boulevard, Milwaukee, WI 53211, USA; kaplan@uwm.edu \\ 2 Department of Astronomy and Astrophysics, University of Toronto, \\ 50 St. George Street, Toronto, ON M5S 3H8, Canada; mhvk@astro.utoronto.ca \\ ${ }^{3}$ Institut für Theoretische Physik und Astrophysik, University of Kiel, D-24098 Kiel, Germany \\ ${ }^{4}$ Department of Physics and Astronomy, University of British Columbia, 6224 Agricultural Road, Vancouver, British Columbia V6T 1Z1, Canada \\ ${ }^{5}$ National Radio Astronomy Observatory, 520 Edgemont Road, Charlottesville, VA 22903-2475, USA \\ ${ }^{6}$ ASTRON, the Netherlands Institute for Radio Astronomy, Postbus 2, 7990 AA Dwingeloo, The Netherlands \\ ${ }^{7}$ Department of Physics, McGill University, 3600 rue University, Montreal, Quebec H3A 2T8, Canada \\ ${ }^{8}$ Astronomical Institute “Anton Pannekoek," University of Amsterdam, Postbus 94249, 1090 GE Amsterdam, The Netherlands \\ ${ }^{9}$ Department of Physics and Astronomy, West Virginia University, White Hall, Box 6315, Morgantown, WV 26506-6315, USA \\ ${ }^{10}$ Physics and Astronomy Department, Western Kentucky University, 1906 College Heights Boulevard 11077, \\ Bowling Green, KY 42101-1077, USA \\ Received 2014 January 4; accepted 2014 January 28; published 2014 February 18
}

\begin{abstract}
The hierarchical triple system PSR J0337+1715 offers an unprecedented laboratory to study secular evolution of interacting systems and to explore the complicated mass-transfer history that forms millisecond pulsars and helium-core white dwarfs. The latter in particular, however, requires knowledge of the properties of the individual components of the system. Here we present precise optical spectroscopy of the inner companion in the PSR $\mathrm{J} 0337+1715$ system. We confirm it as a hot, low-gravity DA white dwarf with $T_{\text {eff }}=15,800 \pm 100 \mathrm{~K}$ and $\log _{10}(g)=5.82 \pm 0.05$. We also measure an inner mass ratio of $0.1364 \pm 0.0015$, entirely consistent with that inferred from pulsar timing, and a systemic radial velocity of $29.7 \pm 0.3 \mathrm{~km} \mathrm{~s}^{-1}$. Combined with the mass $\left(0.19751 M_{\odot}\right)$ determined from pulsar timing, our measurement of the surface gravity implies a radius of $0.091 \pm 0.005 R_{\odot}$; combined further with the effective temperature and extinction, the photometry implies a distance of $1300 \pm 80 \mathrm{pc}$. The high temperature of the companion is somewhat puzzling: with current models, it likely requires a recent period of unstable hydrogen burning, and suggests a surprisingly short lifetime for objects at this phase in their evolution. We discuss the implications of these measurements in the context of understanding the PSR J0337+1715 system, as well as of low-mass white dwarfs in general.
\end{abstract}

Key words: binaries: spectroscopic - pulsars: individual (PSR J0337+1715) - stars: atmospheres stars: neutron - white dwarfs

Online-only material: color figures

\section{INTRODUCTION}

White dwarfs (WDs) are among the best-understood stars, enabling their use as astrophysical tools in investigations of, e.g., the ages and masses of astrophysical systems (e.g., Althaus et al. 2010). However, the lowest-mass WDs with He coresextremely low mass (ELM) WDs with masses $<0.2 M_{\odot}$ (Kilic et al. 2012; Brown et al. 2013 and references therein) - still defy complete understanding, with few reliable independent measurements of masses, sizes and ages known (e.g., van Kerkwijk et al. 1996; Bedin et al. 2005).

Yet, these properties are important for understanding the evolution of ELM WDs and the binaries they are found in (Iben \& Livio 1993; Marsh et al. 1995). For instance, while one would naively expect low-mass WDs to cool quickly, given their relatively large size and small heat capacity, some ELM WDs can remain bright and hot (cf. Lorimer et al. 1995) because they

\footnotetext{
* Based on observations obtained under Program GN-2012B-Q-43 at the Gemini Observatory, which is operated by the Association of Universities for Research in Astronomy, Inc., under a cooperative agreement with the NSF on behalf of the Gemini partnership: the National Science Foundation (United States), the National Research Council (Canada), CONICYT (Chile), the Australian Research Council (Australia), Ministério da Ciência, Tecnologia e Inovação (Brazil) and Ministerio de Ciencia, Tecnología e Innovación Productiva (Argentina).
}

have outer hydrogen layers sufficiently thick for nuclear fusion to continue - stably or otherwise - for several Gyr (Alberts et al. 1996; Serenelli et al. 2002; Panei et al. 2007; Althaus et al. 2013). Improving our understanding of ELM WD cooling would aid in evolutionary models for, e.g., millisecond pulsars and the later stages of mass transfer (e.g., Tauris et al. 2012; Antoniadis et al. 2012, 2013; Kaplan et al. 2013). Similarly, improved masses and radii would aid in determining the final fates of double-WD binaries (Deloye \& Bildsten 2003; Marsh et al. 2004; D'Antona et al. 2006; Kaplan et al. 2012): R CrB stars, AM CVn binaries, or even SNe Ia (Iben \& Tutukov 1984; Webbink 1984).

PSR J0337+1715 (hereafter PSR J0337; Ransom et al. 2014) was discovered in the $350 \mathrm{MHz}$ Green Bank Telescope Driftscan survey (Boyles et al. 2013; Lynch et al. 2013), and initial timing observations found a $2.7 \mathrm{~ms}$ spin period, a 1.6 day orbital period, and a likely companion mass of $0.1-0.2 M_{\odot}$, all of which are consistent with expectations for a fully recycled pulsar with a low-mass He WD companion (van Kerkwijk et al. 2005; Tauris et al. 2012). However, further deviations to the observed spin period soon became apparent, suggesting the presence of an additional body in the system. This was confirmed with an intensive timing campaign, finding an outer orbital period of 327 days (Ransom et al. 2014). By comparing the pulsar's pulse arrival times with numerical integrations of possible orbits, the masses, inclinations, and orbital parameters 
Table 1

Log of Observations and Velocity Measurements

\begin{tabular}{|c|c|c|c|c|c|c|c|c|c|c|}
\hline $\begin{array}{l}\text { Date } \\
\text { None }\end{array}$ & $\begin{array}{c}\text { UT } \\
\text { None }\end{array}$ & $\begin{array}{l}\lambda_{c}{ }^{a} \\
(\AA)\end{array}$ & $\begin{array}{l}\text { Seeing } \\
(\operatorname{arcsec})\end{array}$ & $\begin{array}{c}\text { Shift }^{\mathrm{b}} \\
(\operatorname{arcsec})\end{array}$ & $\begin{array}{l}\text { Offset }^{\mathrm{c}} \\
(\operatorname{arcsec})\end{array}$ & $\begin{array}{c}\text { MJD }_{\text {bar }} \\
\text { None }\end{array}$ & $\begin{array}{c}\phi^{\text {ind }} \\
\text { None }\end{array}$ & $\begin{array}{c}v_{\mathrm{PSR}}^{\mathrm{in}} \mathrm{e} \\
\left(\mathrm{km} \mathrm{s}^{-1}\right)\end{array}$ & $\begin{array}{c}v_{\mathrm{PSR}}^{\text {out f }} \\
\left(\mathrm{km} \mathrm{s}^{-1}\right)\end{array}$ & $\begin{array}{c}v_{\mathrm{WD}}{ }^{\mathrm{g}} \\
\left(\mathrm{km} \mathrm{s}^{-1}\right)\end{array}$ \\
\hline \multirow{6}{*}{2012 Nov 9} & $08: 44$ & 4250 & 1.4 & -0.024 & -0.010 & 56240.3674 & 0.0918 & -10.68 & 4.92 & $117 \pm 4$ \\
\hline & $08: 56$ & 4200 & 1.3 & -0.055 & -0.025 & 56240.3760 & 0.0971 & -11.09 & 4.92 & $112 \pm 5$ \\
\hline & 09:10 & 4250 & 1.3 & -0.055 & -0.025 & 56240.3863 & 0.1034 & -11.55 & 4.92 & $117 \pm 4$ \\
\hline & $09: 24$ & 4200 & 1.2 & -0.057 & -0.029 & 56240.3956 & 0.1091 & -11.96 & 4.92 & $133 \pm 4$ \\
\hline & $11: 32$ & 4250 & 1.9 & 0.034 & 0.009 & 56240.4850 & 0.1640 & -15.00 & 4.92 & $149 \pm 4$ \\
\hline & $11: 46$ & 4200 & 1.9 & 0.036 & 0.009 & 56240.4945 & 0.1698 & -15.22 & 4.92 & $149 \pm 5$ \\
\hline \multirow[t]{4}{*}{2012 Nov 14} & $07: 52$ & 4250 & 1.0 & -0.047 & -0.028 & 56245.3318 & 0.1387 & -13.79 & 4.84 & $128 \pm 4$ \\
\hline & 08:06 & 4200 & 0.9 & -0.047 & -0.030 & 56245.3413 & 0.1445 & -14.10 & 4.84 & $139 \pm 4$ \\
\hline & $12: 22$ & 4250 & 1.8 & -0.021 & -0.006 & 56245.5193 & 0.2537 & -16.08 & 4.84 & $159 \pm 5$ \\
\hline & $12: 36$ & 4200 & 1.8 & 0.017 & 0.005 & 56245.5288 & 0.2596 & -15.98 & 4.84 & $155 \pm 5$ \\
\hline \multirow[t]{4}{*}{2012 Nov 15} & $08: 23$ & 4250 & 1.2 & -0.133 & -0.067 & 56246.3536 & 0.7658 & 15.85 & 4.82 & $-81 \pm 4$ \\
\hline & $08: 37$ & 4200 & 1.5 & -0.131 & -0.050 & 56246.3632 & 0.7717 & 15.70 & 4.82 & $-81 \pm 4$ \\
\hline & 11:02 & 4250 & 1.6 & -0.060 & -0.021 & 56246.4636 & 0.8333 & 12.89 & 4.82 & $-61 \pm 4$ \\
\hline & $11: 15$ & 4200 & 1.5 & -0.050 & -0.020 & 56246.4731 & 0.8392 & 12.51 & 4.82 & $-57 \pm 4$ \\
\hline \multirow[t]{2}{*}{2012 Nov 16} & 08:05 & 4250 & 0.8 & -0.045 & -0.034 & 56247.3409 & 0.3718 & -10.11 & 4.79 & $100 \pm 4$ \\
\hline & 08:19 & 4200 & 0.8 & -0.052 & -0.039 & 56247.3505 & 0.3776 & -9.63 & 4.79 & $100 \pm 4$ \\
\hline \multirow[t]{2}{*}{2012 Dec 15} & $08: 45$ & 4250 & 1.3 & -0.065 & -0.030 & 56276.3683 & 0.1858 & -15.72 & 3.33 & $150 \pm 4$ \\
\hline & $08: 58$ & 4200 & 1.3 & -0.027 & -0.013 & 56276.3772 & 0.1912 & -15.86 & 3.32 & $151 \pm 4$ \\
\hline \multirow[t]{2}{*}{2012 Dec 17} & 10:09 & 4250 & 2.2 & -0.051 & -0.011 & 56278.4272 & 0.4491 & -2.93 & 3.17 & $54 \pm 5$ \\
\hline & $10: 23$ & 4200 & 2.1 & -0.078 & -0.018 & 56278.4367 & 0.4550 & -2.34 & 3.17 & $47 \pm 5$ \\
\hline \multirow[t]{4}{*}{2012 Dec 18} & $04: 54$ & 4250 & 1.2 & 0.011 & 0.005 & 56279.2084 & 0.9285 & 4.98 & 3.11 & $-1 \pm 4$ \\
\hline & 05:08 & 4200 & 1.2 & -0.013 & -0.006 & 56279.2180 & 0.9343 & 4.41 & 3.11 & $-6 \pm 4$ \\
\hline & $08: 16$ & 4250 & 2.4 & -0.115 & -0.021 & 56279.3484 & 0.0144 & -3.71 & 3.10 & $52 \pm 5$ \\
\hline & $08: 29$ & 4200 & 2.2 & -0.177 & -0.036 & 56279.3580 & 0.0202 & -4.29 & 3.10 & $60 \pm 5$ \\
\hline \multirow[t]{6}{*}{2012 Dec 19} & 05:07 & 4250 & 1.2 & 0.075 & 0.036 & 56280.2176 & 0.5477 & 6.92 & 3.03 & $-27 \pm 4$ \\
\hline & $05: 21$ & 4200 & 1.3 & 0.070 & 0.032 & 56280.2272 & 0.5535 & 7.46 & 3.03 & $-26 \pm 4$ \\
\hline & $07: 16$ & 4250 & 1.6 & 0.114 & 0.041 & 56280.3068 & 0.6024 & 11.48 & 3.02 & $-55 \pm 4$ \\
\hline & $07: 30$ & 4200 & 1.9 & 0.119 & 0.033 & 56280.3164 & 0.6083 & 11.90 & 3.02 & $-55 \pm 5$ \\
\hline & $10: 45$ & 4250 & 1.1 & -0.054 & -0.030 & 56280.4519 & 0.6914 & 15.86 & 3.01 & $-78 \pm 4$ \\
\hline & $10: 58$ & 4200 & 1.0 & -0.089 & -0.054 & 56280.4614 & 0.6973 & 15.99 & 3.01 & $-74 \pm 3$ \\
\hline \multirow[t]{3}{*}{ 2013 Jan 18} & $07: 33$ & 4250 & 1.3 & 0.004 & 0.002 & 56310.3181 & 0.0074 & -4.34 & 0.19 & $63 \pm 5$ \\
\hline & $07: 46$ & 4200 & 1.5 & -0.032 & -0.012 & 56310.3272 & 0.0130 & -4.88 & 0.19 & $69 \pm 6$ \\
\hline & $07: 58$ & 4200 & 1.5 & -0.032 & -0.012 & 56310.3357 & 0.0182 & -5.38 & 0.19 & $70 \pm 5$ \\
\hline \multirow[t]{2}{*}{2013 Feb 05} & $05: 33$ & 4250 & 1.6 & -0.025 & -0.008 & 56328.2349 & 0.9810 & -3.92 & -1.61 & $55 \pm 4$ \\
\hline & $05: 46$ & 4200 & 1.6 & -0.030 & -0.010 & 56328.2438 & 0.9864 & -4.45 & -1.62 & $67 \pm 4$ \\
\hline
\end{tabular}

Notes.

${ }^{\text {a }}$ Center wavelength of each observation.

${ }^{b}$ Deviation of the target from the center of the slit in the acquisition image.

${ }^{c}$ Deviation of the center of light from the center of the slit, calculated using the FWHM measured from the spectra.

d Orbital phase for the inner orbit.

e Inferred velocity for the pulsar due to the inner orbit relative to the system barycenter.

${ }^{\mathrm{f}}$ Inferred velocity for the pulsar due to the outer orbit relative to the system barycenter

g Measured velocity for the white dwarf, with all corrections applied.

of the system could be determined precisely (Ransom et al. 2014; A. M. Archibald et al. 2014, in preparation), with a pulsar mass of $1.4378 \pm 0.0013 M_{\odot}$, an inner companion mass of $0.19751 \pm 0.0015 M_{\odot}$, and an outer companion mass of $0.4101 \pm 0.0003 M_{\odot}$; both inner and outer orbits are inclined by about $39^{\circ}$ to the plane of the sky.

Before the nature of the system was fully determined, we identified an unusually blue object coincident with it, which, based on initial spectroscopy and photometry, we identified as the inner companion, almost certainly a hot low-mass WD (Ransom et al. 2014). The brightness of the system, its proximity, and the detailed constraints offered by pulsar timing make the system a fantastic laboratory to explore the atmosphere, structure, and evolution of ELM WDs. Here, we present an intensive spectroscopic campaign aimed to do so, which also serves as a valuable cross-check of the pulsar timing results.

\section{OBSERVATIONS}

Spectra of the counterpart of PSR J0337 were taken for us between 2012 November and 2013 January with the Gemini Multi Object Spectrograph (GMOS; Hook et al. 2004) of the GeminiNorth telescope (see Table 1). We used the 1200 line $\mathrm{mm}^{-1}$ grating, covering the 3500-5000 A range with the three $2048 \times 4608$ pixel CCD detectors (which were binned $4 \times 4$, giving a spatial scale of 0 '.29 pixel $^{-1}$ and a dispersion of $0.94 \AA$ pixel $^{-1}$ ). As our object is relatively bright and has broad absorption lines, we could use poor-seeing conditions and hence opted for a wide, $1^{\prime \prime} .5$ slit. With the typical seeing of $1^{\prime \prime} .2$, and given the anamorphic plate scale of 0 !'38 pixel $^{-1}$ (for our grating setting; Murowinski et al. 2003), the resolution is $\sim 3 \AA$.

In each visit, we took two 10 minute exposures offset by $50 \AA$ to cover the gaps between the detectors. Between the exposures, we took incandescent and CuAr lamp spectra for 
both settings, and before and after we took images through the slit to be able to constrain velocity offsets due to centering errors. For flux calibration, spectra were taken on a photometric night using the same settings through a $5^{\prime \prime}$ slit, immediately followed by exposures of the WD spectrophotometric standard GD 71 (Bohlin \& Gilliland 2004).

We oriented the slit to include another object on the slit, hoping to use it as a local flux and velocity reference. Unfortunately, the first object we picked-the only relatively bright one nearby, at a separation of $27^{\prime \prime} .7$ and position angle 8.3 - turned out to be a galaxy, which is not useful as it fills the slit. Hence, after the first set of data, we tried another object (at 58", $-112^{\circ}$ ) which was fainter but which we hoped would have sufficiently narrow lines to still be useful. This, however, turned out to be a quasar. Hence, as will be clear below, our final velocity uncertainties have a significant component due to slit centroiding errors.

We reduced the data using custom python scripts. First, we subtracted bias levels as determined from overscan regions for each of the six read-out channels, divided by their gains to give electron counts (with gains adjusted to ensure counts for flat fields were consistent between different read-out channels), combined detector halves, and divided by normalized flat fields. The spectra were extracted optimally, fitting at each dispersion position the trace of the two objects with a Moffat function of the form $\left(1+\left[\left(x-x_{c}\right) / w\right]^{2}\right)^{-\beta}$ and the sky with a seconddegree polynomial (with the trace position $x_{c}$ and width $w$ allowed to vary slowly with wavelength, and the exponent fixed to $\beta=2.5$ ).

For wavelength calibration, we first obtained accurate calibrations for a set of daytime $\mathrm{CuAr}$ spectra taken through a narrow, 0.5 slit, in which many fewer lines are blended. Fitting a third-order polynomial for wavelength as a function of detector position, optimizing simultaneously for the offsets between the chips, we find root-mean-square residuals of $0.017 \AA$ (for 79 lines; relative to copper and argon line wavelengths from the NIST database). For each nighttime arc, we measure the shift relative to the daytime arc taken at the same setting, and then apply the daytime calibration.

The spectra were flux-calibrated in three steps. First, all spectra were corrected approximately for atmospheric extinction using the average Mauna Kea extinction curve from Buton et al. (2013). Next, for the narrow-slit spectra, slit and cloud losses were measured by fitting a quadratic function to the count-rate ratio with the wide-slit observation. Finally, we divided by the instrumental response derived from the smoothed ratio of the count rates and fluxes of GD 71. While the wavelength region we covered does not fully overlap any of the photometric filters, extrapolating the short-wavelength part of the spectrum using a power-law gives a good agreement (better than $1 \sigma$ ) with the measured Sloan Digital Sky Survey $u^{\prime}$ photometry.

\section{ANALYSIS}

\subsection{Velocities}

We determined velocities by fitting a template to the data for a range of trial velocities, at each allowing for normalization and possible variations with wavelength using a linear function. For the template, we used a pure hydrogen model atmosphere with $T_{\text {eff }}=15,800 \mathrm{~K}$ and $\log _{10}(g)=5.80$, close to the bestfit parameters determined below (Section 3.2), convolving it with a Gaussian with a width set by the typical seeing of $1^{\prime \prime} .2$ (equivalent to $2.9 \AA$ ), and truncated at $1^{\prime \prime} .5$ to mimic the slit. The fits to the spectra were good, with typical $\chi^{2}=1440$ for 1527

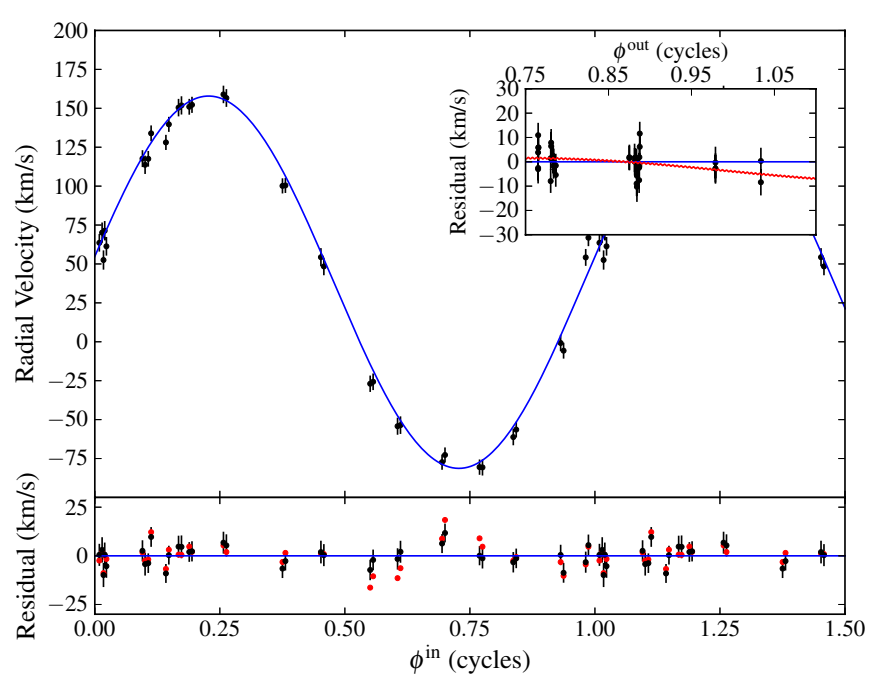

Figure 1. Radial velocities of the optical counterpart to PSR J0337. Main panel: observed velocities plus the velocity expected for the outer orbit, as a function of the phase of the inner orbit (repeated 1.5 times for clarity), with the best-fit model overdrawn (solid line). Lower panel: residuals with respect to that model with and without correction for small slit-centering errors (black and red points, respectively). Inset: residuals as a function of outer orbital phase, with inner velocity contribution removed, and with models with and without the outer object overdrawn (blue and red solid lines, respectively).

(A color version of this figure is available in the online journal.)

degrees of freedom, and implied formal velocity uncertainties of $\sim 4 \mathrm{~km} \mathrm{~s}^{-1}$.

An additional uncertainty in our velocities is the extent to which the object was properly centered in the slit. As mentioned, we had hoped to use a comparison star to calibrate this, but this turned out to be a quasar. Inspecting the images taken through the slit before and after the spectra, we find that the star has root-mean-square offsets from the slit center of $00^{\prime} 07$, with the largest deviations about twice that (i.e., up to 2 unbinned pixels). The effect on velocity also depends on seeing (for very bad seeing, the slit is better filled and the effect minimized). From the acquisition images themselves, we find flux-weighted offsets about half as large, of 0!.045 (rms). The shifts for the spectra are slightly smaller, since the traces in the spectra show slightly larger seeing, presumably because of jitter in the telescope pointing. Using the centroiding positions from the acquisition images combined with the seeing from the spectral traces, we infer flux-weighted offsets of $0^{\prime \prime} .034$ (rms), corresponding to wavelength shifts of $0.060 \AA$ and, for an assumed effective wavelength of $4200 \AA$, velocity shifts of $\sim 4.3 \mathrm{~km} \mathrm{~s}^{-1}$. In Table 1 , we list the velocities corrected for these shifts and corrected to the solar system barycenter.

To determine the orbit, we fit the velocities to a model $v_{\mathrm{WD}}(t)=\gamma-\left(1 / q_{I}\right) \times v_{\mathrm{PSR}}^{\text {in }}(t)+v_{\mathrm{PSR}}^{\text {out }}(t)$, where $\gamma$ is the systemic radial velocity, $q_{I} \equiv M_{\mathrm{WD}} / M_{\mathrm{PSR}}$ is the inner mass ratio, $v_{\mathrm{PSR}}^{\mathrm{in}}$ is the radial velocity of the pulsar in the inner orbit, and $v_{\mathrm{PSR}}^{\text {out }}$ is the radial velocity of the inner-orbit barycenter in the outer orbit, both as inferred from the timing model (with radialvelocity amplitudes $K_{\mathrm{PSR}}^{\mathrm{in}}=16.291$ and $K_{\mathrm{PSR}}^{\text {out }}=4.978 \mathrm{~km} \mathrm{~s}^{-1}$, respectively). Such a model is traditional for pulsar binaries, where the period and pulsar's velocity are known but the mass ratio is not. For PSR J0337, we do know $q_{I}$, but the analysis serves as a valuable check of the full timing model. The velocities fit reasonably well (Figure 1), although the fit is formally unacceptable, with $\chi^{2}=55.9$ for 33 degrees of freedom ( 35 spectra, 2 parameters), likely because of remaining 


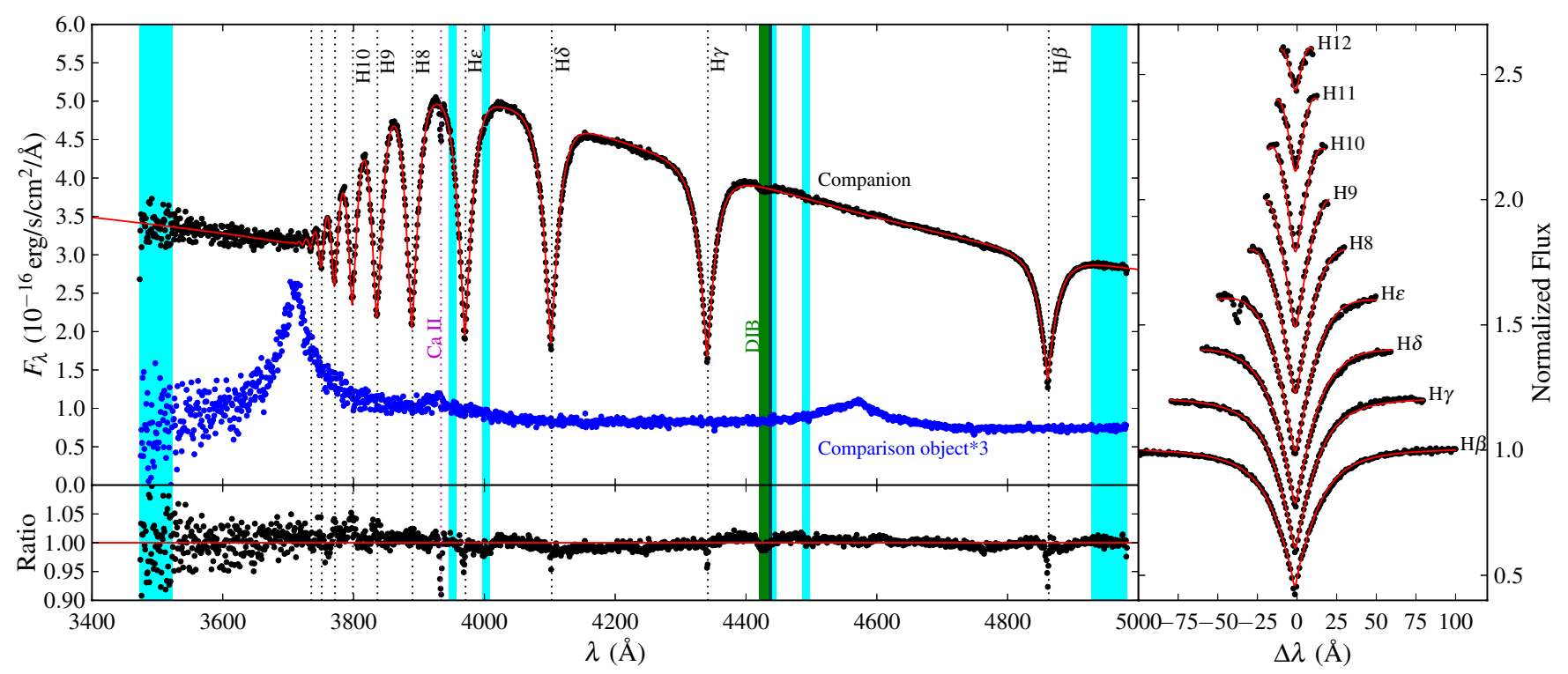

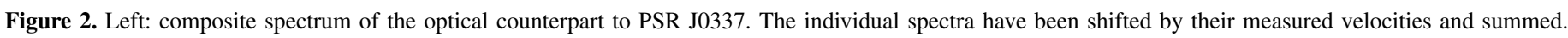

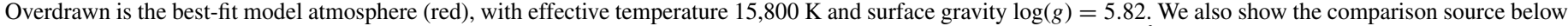

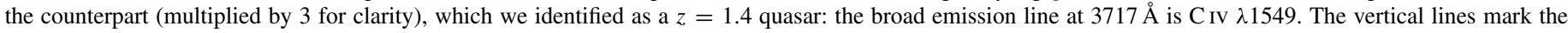

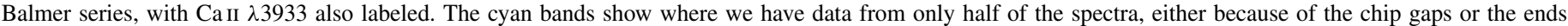

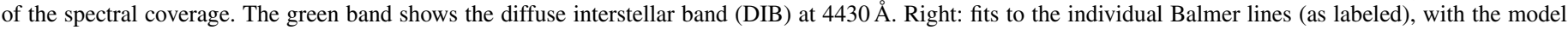

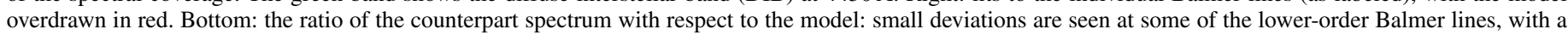
more significant deviation at the Ca II $\lambda 3933$ line.

(A color version of this figure is available in the online journal.)

uncertainties in the slit centering (e.g., due to differential atmospheric refraction). In order to account for this in our parameter uncertainties, we added an additional uncertainty of $3.3 \mathrm{~km} \mathrm{~s}^{-1}$ to the velocity errors (in quadrature), giving a reduced $\chi^{2}$ of 1.0. With that, we find $\gamma=29.7 \pm 0.9 \mathrm{~km} \mathrm{~s}^{-1}$ and $q_{I}=0.1364 \pm 0.0015$, implying $K_{\mathrm{WD}}^{\text {in }}=119.4 \pm 1.3 \mathrm{~km} \mathrm{~s}^{-1}$. This is fully consistent with the value measured from pulsar timing (Ransom et al. 2014): $q_{I}=0.13737 \pm 0.00004$.

For completeness, we note that our results do not depend on whether we include the centroiding shifts, although the fit becomes substantially worse if we do not $\left(\chi^{2}=115\right)$. The fit also does not depend on whether we took the centroid from the nearest acquisition image, or rather interpolated between images taken before and after the spectra. Furthermore, if we leave the prefactor for the centroiding shift free, i.e., include an additional term $\alpha \Delta v$ in the fit, we find $\alpha=1.13 \pm 0.16$. Finally, if we ignore the contribution from the outer orbit, we find $\chi^{2}=58.5$, i.e., the outer orbit is detected marginally even in our velocities.

\subsection{Model Atmosphere Fits}

Given the velocities determined above, we created a composite summed spectrum (Figure 2) by shifting each of the individual measurements back to zero velocity. We see strong Balmer lines, as well as a weaker Ca II $\lambda 3933$ absorption line and some broad absorption near $4430 \AA$ associated with a diffuse interstellar band (DIB).

Metal lines are occasionally seen from WDs (Gänsicke et al. 2012), especially those with low gravities $(<5.6$; Kilic et al. 2012; Kaplan et al. 2013), and they are commonly interpreted as signs of accretion. However, Ca II absorption can also be interstellar in origin. We compared the velocity centroid and width of the $\mathrm{Ca}$ II line using both the spectra corrected for the motion of the WD and only corrected for the motion of the
Earth around the solar system barycenter. We find that the line is marginally narrower when not correcting for the motion of the WD (the depth increases from $9.3 \pm 0.6 \%$ to $10.2 \pm 0.6 \%$ ), suggesting that it is interstellar in this case. This is confirmed by a line of similar strength that we see in the spectrum of the comparison quasar, although the signal-to-noise is lower. The centroid of the $\mathrm{Ca}$ II line as determined from the uncorrected data is $44 \pm 6 \mathrm{~km} \mathrm{~s}^{-1}$, implying a systemic radial velocity of the pulsar relative to the interstellar medium of $-14 \pm 6 \mathrm{~km} \mathrm{~s}^{-1}$; this is not very different from the expectations using the Brand \& Blitz (1993) rotation curve for a distance of $1.3 \mathrm{kpc}$ (see below), where the radial velocity goes between 0 and $-4 \mathrm{~km} \mathrm{~s}^{-1}$ along the line-of-sight. Similarly, with data only corrected for the Earth's motion (and excluding data where the gap between the green and red CCDs came near the DIBs), we measure a depth at the center of the DIB of $2.0 \pm 0.2 \%$. Based on the empirical relation of Krelowski et al. (1987), we infer an extinction $A_{V} \approx 0.3 \mathrm{mag}$.

We next fit for the atmospheric parameters for the WD by comparing against pure hydrogen models computed by one of us (D. Koester). These models covered $T_{\text {eff }}=15,500-16,500 \mathrm{~K}$ in steps of $100 \mathrm{~K}$ and $\log _{10}(g)=5.50-6.50$ in steps of 0.1 dex. We identified some line-free regions to fit a cubic polynomial that represented the difference in normalization between the models and the data, and computed the $\chi^{2}$ of each model with respect to the data. The models were convolved with a function to represent the slit and the average seeing (1".2), as discussed above. We excluded the region around the Ca II $\lambda 3933$ line and $\mathrm{H} \epsilon$ (which is blended with Ca II $\lambda 3968$ ). Overall, our initial fit has $T_{\text {eff }}=15,780 \mathrm{~K}$ and $\log _{10}(g)=5.82$. This fit has $\chi^{2}=4098.2$ for 1613 degrees of freedom, so it is formally unacceptable. Much of the deviation comes from the cores of the lower-order Balmer lines (Figure 2, lower panel). These deviations might be indicative of the outer member of the binary (i.e., third light). However, we find that they track the WD's orbit, 
so are likely just errors in our model or calibration. In particular, NLTE effects might be important; in higher-gravity WDs, they cause deeper cores for $\mathrm{H} \alpha$ and $\mathrm{H} \beta$ at these temperatures.

Computing the best-fit model for each individual observation gave similar results, with $T_{\text {eff }}=15,882 \pm 35 \mathrm{~K}$ and $\log _{10}(g)=$ $5.85 \pm 0.01$, where the uncertainties are the formal errors in the means. To account for the formally poor fit and model uncertainties, we increase the uncertainties to \pm 0.05 dex and $\pm 100 \mathrm{~K}$, which are about the smallest we would believe for an object in this relatively unconstrained part of the WD cooling sequence. We therefore adopt as our best-fit model $T_{\text {eff }}=15,800 \pm 100 \mathrm{~K}$ and $\log _{10}(g)=5.82 \pm 0.05$. For this effective temperature, the best-fit extinction based on the photometry is $A_{V}=0.44 \pm 0.04 \mathrm{mag}$ (Ransom et al. 2014).

\section{DISCUSSION AND CONCLUSIONS}

Our measurements provide the velocities and atmospheric parameters of the inner WD in the PSR J0337 system. The velocities serve to confirm the more precise ones inferred from pulsar timing. In addition, they show that the systemic velocity is low. This is not unexpected, since any kick imparted to the system in the supernova explosion that formed the neutron star must have been small for the triple to survive (Tauris \& van den Heuvel 2014). One thus expects the proper motion to be similarly small.

Our measurement of the surface gravity, combined with the precise mass from timing, implies a radius of $0.091 \pm$ $0.005 R_{\odot}$. Combining this in turn with the effective temperature, extinction, and photometry, one infers a distance of $1300 \pm 80 \mathrm{pc}$ (Ransom et al. 2014). With an accurate parallax from very-long baseline interferometry (measurements are in progress), this can be used to infer the surface gravity and thus test the model atmospheres in an otherwise poorly constrained regime.

The mass and radius of the inner WD are consistent with those expected for a young, low-mass helium-core WD, similar to the WD companions found in other binaries (Section 1). Compared to low-mass WDs around pulsars, ${ }^{11}$ however, the source stands out for being hotter than most. This must be intrinsic as possible contributions from pulsar irradiation and tidal heating are negligible.

The high temperature is surprising as it would suggest the system is in a short-lived state and hence that similar systems are common-which, empirically, they are not. This suggestion arises because in current evolutionary models of helium WDs, temperatures in excess of $\sim 12,000 \mathrm{~K}$ are only achieved in models with unstable shell flashes (e.g., Driebe et al. 1998; Althaus et al. 2001). In those shell flashes, however, most of the thick hydrogen layer is lost, and hence the WD will cool relatively quickly. Furthermore, while the flashing state may last 200 Myr (Althaus et al. 2013), the typical cooling timescales at these temperatures are short, a few $10 \mathrm{Myr}$ for each flash, and hence the total time spent at high temperatures is often $<100 \mathrm{Myr}$, depending on mass. These timescales are still far longer than the expected sedimentation timescale for helium ( $\sim 10^{3} \mathrm{yr}$ ) following mixing during a shell flash, consistent with the lack of any He I in the spectrum of the inner WD (cf. Kaplan et al. 2013). Based on inspection, we can roughly limit its abundance to $10^{-2.5} \mathrm{H}$ (by number), which would not change our inferred $\log _{10}(g)$ by more than our quoted uncertainty. If the

\footnotetext{
11 One cannot easily compare low-mass white dwarfs with white-dwarf or A-star companions, since for those systems there are strong observational biases to find hotter, more luminous, and larger white dwarfs.
}

inner WD is really only a few 100 Myr old, it almost certainly formed last, as also expected from simple models (Ransom et al. 2014; Tauris \& van den Heuvel 2014), although we cannot strictly exclude the opposite: at the upper limit to the temperature of the outer WD of 20,000 K (Ransom et al. 2014), the cooling time would be 30-100 Myr.

Of course, it could be a coincidence that we found such a hot WD companion. However, a similarly hot companion was found for PSR J1816+4510 (Kaplan et al. 2013). Since typical millisecond pulsars remain visible for a Hubble time, and since we know $\sim 50$ pulsars with low-mass WD companions, this suggests that WDs can stay hot for $~ 500 \mathrm{Myr}$, substantially longer than expected in current theoretical models. The discrepancy is made worse by the fact that WDs with mass below $\lesssim 0.18 M_{\odot}$ are not expected to get this hot at all (they should not have flashes). Observationally, however, lifetimes of a few hundred Myr seem also indicated by the prevalence of hot, low-mass WDs around A stars (which have ages of $\lesssim 1 \mathrm{Gyr}$ ).

We thank an anonymous referee for a helpful suggestion. We thank Gemini staff Atsuko Nitta, John Blakeslee, and Kristin Chiboucas for helping assure the high quaility of these data. The National Radio Astronomy Observatory is a facility of the National Science Foundation, operated under cooperative agreement by Associated Universities, Inc. Balmer/Lyman lines in the models were calculated with the modified Stark broadening profiles of Tremblay \& Bergeron (2009), kindly made available by the authors. M.H.v.K. and I.H.S. acknowledge funding from NSERC Discovery Grants. J.W.T.H. and A.M.A. acknowledge support from a Vrije Competitie grant from NWO. We made extensive use of SIMBAD, ADS, and Astropy (http://www.astropy.org; Astropy Collaboration et al. 2013).

Facility: Gemini:Gillett (GMOS)

\section{REFERENCES}

Alberts, F., Savonije, G. J., van den Heuvel, E. P. J., \& Pols, O. R. 1996, Natur, 380,676

Althaus, L. G., Córsico, A. H., Isern, J., \& García-Berro, E. 2010, A\&ARv, 18,471

Althaus, L. G., Miller Bertolami, M. M., \& Córsico, A. H. 2013, A\&A, 557, A19

Althaus, L. G., Serenelli, A. M., \& Benvenuto, O. G. 2001, MNRAS, 323, 471 Antoniadis, J., Freire, P. C. C., Wex, N., et al. 2013, Sci, 340, 448

Antoniadis, J., van Kerkwijk, M. H., Koester, D., et al. 2012, MNRAS, 423, 3316

Astropy Collaboration, Robitaille, T. P., Tollerud, E. J., et al. 2013, A\&A, 558, A33

Bedin, L. R., Salaris, M., Piotto, G., et al. 2005, ApJL, 624, L45

Bohlin, R. C., \& Gilliland, R. L. 2004, AJ, 128, 3053

Boyles, J., Lynch, R. S., Ransom, S. M., et al. 2013, ApJ, 763, 80

Brand, J., \& Blitz, L. 1993, A\&A, 275, 67

Brown, W. R., Kilic, M., Allende Prieto, C., Gianninas, A., \& Kenyon, S. J. 2013, ApJ, 769, 66

Buton, C., Copin, Y., Aldering, G., et al. 2013, A\&A, 549, A8

D’Antona, F., Ventura, P., Burderi, L., \& Teodorescu, A. 2006, ApJ, 653, 1429 Deloye, C. J., \& Bildsten, L. 2003, ApJ, 598, 1217

Driebe, T., Schoenberner, D., Bloecker, T., \& Herwig, F. 1998, A\&A, 339, 123 Gänsicke, B. T., Koester, D., Farihi, J., et al. 2012, MNRAS, 424, 333

Hook, I. M., Jørgensen, I., Allington-Smith, J. R., et al. 2004, PASP, 116, 425 Iben, I., Jr., \& Livio, M. 1993, PASP, 105, 1373

Iben, I., Jr., \& Tutukov, A. V. 1984, ApJS, 54, 335

Kaplan, D. L., Bhalerao, V. B., van Kerkwijk, M. H., et al. 2013, ApJ, 765, 158 Kaplan, D. L., Bildsten, L., \& Steinfadt, J. D. R. 2012, ApJ, 758, 64

Kilic, M., Brown, W. R., Allende Prieto, C., et al. 2012, ApJ, 751, 141

Krelowski, J., Walker, G. A. H., Grieve, G. R., \& Hill, G. M. 1987, ApJ, 316,449

Lorimer, D. R., Lyne, A. G., Festin, L., \& Nicastro, L. 1995, Natur, 376, 393 
Lynch, R. S., Boyles, J., Ransom, S. M., et al. 2013, ApJ, 763, 81

Marsh, T. R., Dhillon, V. S., \& Duck, S. R. 1995, MNRAS, 275, 828

Marsh, T. R., Nelemans, G., \& Steeghs, D. 2004, MNRAS, 350, 113

Murowinski, R. G., Allington-Smith, J. R., Beard, S. M., et al. 2003, Proc. SPIE, 4841,1189

Panei, J. A., Althaus, L. G., Chen, X., \& Han, Z. 2007, MNRAS, 382, 779

Ransom, S. M., Stairs, I. H., Archibald, A. M., et al. 2014, Natur, 505, 520

Serenelli, A. M., Althaus, L. G., Rohrmann, R. D., \& Benvenuto, O. G. 2002, MNRAS, 337, 1091
Tauris, T. M., Langer, N., \& Kramer, M. 2012, MNRAS, 425, 1601

Tauris, T. M., \& van den Heuvel, E. P. J. 2014, ApJ, 781, 13

Tremblay, P.-E., \& Bergeron, P. 2009, ApJ, 696, 1755

van Kerkwijk, M. H., Bassa, C. G., Jacoby, B. A., \& Jonker, P. G. 2005, in ASP Conf. Ser. 328, Binary Radio Pulsars, ed. F. A. Rasio \& I. H. Stairs (San Fransisco, CA: ASP), 357

van Kerkwijk, M. H., Bergeron, P., \& Kulkarni, S. R. 1996, ApJL, 467, L89

Webbink, R. F. 1984, ApJ, 277, 355 